\title{
Atom-atom structure factors of hydrogen halides: A molecular approach revisited
}

Cite as: J. Chem. Phys. 103, 3680 (1995); https://doi.org/10.1063/1.470044

Submitted: 03 April 1995. Accepted: 31 May 1995. Published Online: 31 August 1998

M. Alvarez, E. Lomba, C. Martín, and M. Lombardero

ARTICLES YOU MAY BE INTERESTED IN

Comparison of simple potential functions for simulating liquid water

The Journal of Chemical Physics 79, 926 (1983); https://doi.org/10.1063/1.445869

\section{Lock-in Amplifiers up to $600 \mathrm{MHz}$}
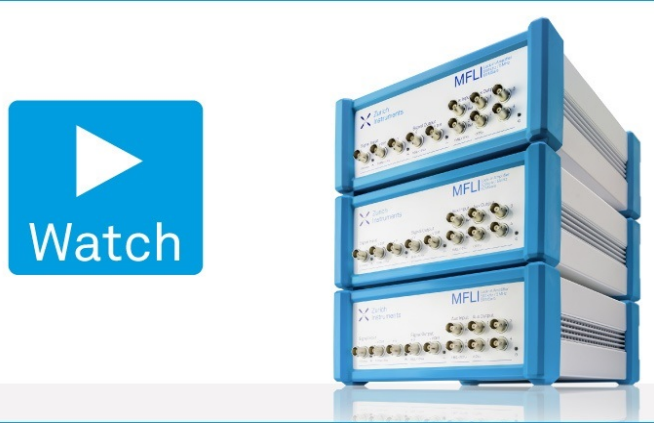

J. Chem. Phys. 103, 3680 (1995); https://doi.org/10.1063/1.470044 


\title{
Atom-atom structure factors of hydrogen halides: A molecular approach revisited
}

\author{
M. Alvarez and E. Lomba \\ Instituto de Química Física Rocasolano, CSIC, Serrano 119, E-28006 Madrid, Spain \\ C. Martín and M. Lombardero \\ Instituto de Química Física Rocasolano, CSIC, Serrano 119, E-28006 Madrid \\ and Departamento Química Física I, U. Complutense, E-28040 Madrid, Spain
}

(Received 3 April 1995; accepted 31 May 1995)

\begin{abstract}
The aim of this paper is to explore the possibilities of a novel molecular integral equation approach to produce information on the atom-atom microscopic structure of molecular fluids via multidimensional integration of the molecular pair distribution function. In particular, atom-atom structure factors for hydrogen halides $(\mathrm{HCl}$ and $\mathrm{HI})$ are computed from the integral equation for heteronuclear fluids modeled by a two-center Lennard-Jones potential with and without multipole terms. Theoretical results are compared both with experimental partial structure factors and computer simulation results. Theory and simulation agree remarkably well both for thermodynamics and microscopic structure. The comparison with experimental partial structure factors is satisfactory within the limitations due to the rough modeling used for describing the real fluid. (C) 1995 American Institute of Physics.
\end{abstract}

\section{INTRODUCTION}

The work presented herein is part of an ongoing project concerning the theoretical analysis of molecular fluids within the framework of the Molecular Integral Equation theory. The core of it is devoted to an accurate calculation of the site-site correlation functions, either $g_{\alpha \beta}(r)$ or its Fouriertransformed function, the atomic structure factor,

$$
S_{\alpha \beta}(Q)-\delta_{\alpha \beta}=\rho \int\left[g_{\alpha \beta}(r)-1\right] \exp (i \mathbf{Q} \mathbf{r}) d \mathbf{r},
$$

which is susceptible to be directly compared with corresponding experimental quantities obtained by means of both neutron and x-ray diffraction measurements.

The molecular integral equation approach employed herein for the description of thermodynamics and microscopic structure of the molecular fluid has already been presented in detail in a series of recent papers. ${ }^{1-3}$ Nevertheless, for completeness it will be briefly sketched in Sec. II. In short, the approximation reduces to the molecular OrnsteinZernike equations (OZ) coupled with a Reference Hypernetted Chain closure (RHNC) ${ }^{4,5}$ and with a parametrized harddumbbell reference bridge function, determined in the modified Verlet's approximation. ${ }^{6}$ This approach will be throughout the paper denoted by RHNC-VM.

Up to now, the RHNC-VM approximation has been successfully implemented to study a variety of diatomic fluids, ${ }^{1,2,7}$ but atomic structure factors, $S_{\alpha \beta}(Q)$, have only been determined on the basis of the calculation of the molecular pair distribution function, $g(12)$, using a reference frame with the $z$ axis connecting the atomic sites of two different molecules, which enables the computation of the site-site correlation functions as the plain orientational average of the molecular pair distribution function. The procedure, though simple, has severe drawbacks as soon as the rewarding homonuclear symmetry is broken. Thus, for a simple heteronuclear system, the integral equation would have to be solved for each choice of the reference frame, i.e., for each site-site distribution desired. Moreover, the bizarre reference frame required to compute the unlike correlations further breaks the symmetry simplifications within the expansion of the molecular pair distribution function. Therefore, the new calculations presented here are based on the integration of the molecular distribution function, by means of a simple procedure explained in depth in Sec. II. In any case, the excellent agreement obtained for liquid halogens in contrast with the discrepancies of other approximations like the site-site Integral Equation approach (RISM), showed unambiguously the ability of the RHNC-VM to yield accurate site-site distribution functions. ${ }^{7}$

These results encouraged us to proceed further and the obvious next step should then focus on heteronuclear diatomic fluids. Therefore we have concentrated our study here on liquid hydrogen halides, particularly $\mathrm{HCl}$ and $\mathrm{HI}$, the only two systems for which experimental data are available. In spite of the fact, this kind of liquids constitutes one of the simplest molecular fluids, the knowledge of their intermolecular interactions is far from being satisfactory. Actually, so far only a few simulation studies of liquid $\mathrm{HCl}^{8-10}$ were performed years ago, modeling the intermolecular interaction by means of an 2CLJ plus point multipoles, and there seems to be no simulation studies available for liquid HI. An additional problem is the scarcity of experimental data to be used as benchmark of theoretical and simulation results. Only a couple of neutron diffraction measurements have been reported concerning $\mathrm{HCl}$ liquid $^{11,12}$ and one regarding $\mathrm{HI}$ liquid. ${ }^{13}$ To our knowledge no x-ray diffraction measurements have been reported for these systems. For these systems we have explored several simple potential models, both using the above-mentioned integral equation and Monte Carlo computer simulation.

The rest of the paper can be sketched as follows. Section II contains the essentials of the theoretical approach, and, in 
particular, the details of the determination of the atom-atom distribution function from the molecular distribution function. The method to extract the partial structure factors from the experimental molecular structure factor obtained by neutron diffraction measurements will be outlined in Sec. III. Finally, in Sec. IV the most significant results of this work are presented and analyzed.

\section{THEORETICAL CONSIDERATIONS}

The RHNC-VM approximation reduces to the molecular $\mathrm{OZ}$ equation, which for fluids composed of linear particles reads as

$$
\gamma(12)=\frac{\rho}{4 \pi} \int c(13)[c(32)+\gamma(32)] d \mathbf{3},
$$

coupled with the RHNC closure relation,

$$
c(12)=\exp \left[-\beta u(12)+\gamma(12)-B_{0}(12)\right]-\gamma(12)-1,
$$

where $\gamma(12)=g(12)-1-c(12)$ is the indirect correlation function and $c(12)$ is the direct correlation function. $g(12)$ will be hereinafter denoted also by $g\left(\mathbf{R}_{12}, \omega_{1}, \omega_{2}\right)$ to stress its orientational dependence. This molecular orientational pair distribution function is related to the intermolecular interaction, $u(12)$, and provides the most complete information of the local structure in terms of the pairs of molecules. $\rho$ is the molecular number density and $\beta=\left(k_{B} T\right)^{-1}$. $B_{0}(12)$ is the bridge function of a given reference system. In this case, $B_{0}(12)$ is calculated for a heteronuclear hard diatomic fluid, whose structure is defined in the VM approximation, i.e.,

$$
B_{0}(12)=\frac{1}{2} \frac{\gamma_{0}^{2}(12)}{1+\xi\left(T^{*}, \rho^{*}\right) \gamma_{0}(12)},
$$

with

$$
\xi\left(T^{*}, \rho^{*}\right)=1-\frac{1}{3} \rho^{*} d^{* 3}\left(T^{*}, \rho^{*}\right) .
$$

Here $\gamma_{0}(12)$ is the reference fluid indirect correlation function that satisfies an OZ equation analogous to Eq. (3), but with $B_{0}(12)$ defined by Eq. (4). $T^{*}=k_{B} T / \varepsilon_{a}, \rho^{*}=\rho \sigma_{a}^{3}$, where $\gamma=\sigma_{b} / \sigma_{a}$ and $\varepsilon_{\alpha}$ and $\sigma_{\alpha}$ refer to 2CLJ potential parameters of the largest atom. $d^{*}$ is the equivalent hard sphere diameter. This quantity can be expressed in reduced units as

$$
\begin{aligned}
d^{* 3}= & 1 / 2\left[\left(1+\gamma_{R}^{3}\right)+3 / 2 L^{*}\left(1+\gamma_{R}^{2}\right)-L^{* 3}\right. \\
& \left.+3 / 16 \frac{\left(1-\gamma_{R}^{2}\right)^{2}}{L^{*}}\right],
\end{aligned}
$$

with $d_{a}^{*}=d_{a} / \sigma_{a}, \gamma_{R}=d_{b} / d_{a}$, and $L^{*}$ is the reduced elongation of the reference system, assumed equal to that of the "real" fluid, i.e., $L / \sigma_{a}$. The hard-sphere diameters can be parametrized in terms of the $\mathrm{LJ}$ interaction parameters by ${ }^{3}$

$$
\begin{aligned}
d_{\alpha}= & \sigma_{\alpha} g(\gamma)\left[25-\ln \left(k_{B} T / \varepsilon_{\alpha}\right)\right]\left[25+\ln \left(\rho \sigma_{\alpha}^{3}\right)\right] ; \\
& (\alpha=a, b),
\end{aligned}
$$

$$
g(\gamma)=\alpha\left[1-h\left(\frac{1-\gamma}{1+\gamma}\right)^{2}\right]
$$

with $\alpha=1.62 \times 10^{-3}, h=0.88$. The cross-interaction parameters, $\varepsilon_{a b}$ and $\sigma_{a b}$, are usually calculated using the LorentzBerthelot mixing rules,

$$
\varepsilon_{a b}=\left(\varepsilon_{a} \varepsilon_{b}\right)^{1 / 2} ; \quad \sigma_{a b}=\frac{1}{2}\left(\sigma_{a}+\sigma_{b}\right) .
$$

Thus, according with the additivity of the interaction range parameters in the real system, $d_{a b}=\left(d_{a a}+d_{b b}\right) / 2$ is generally assumed.

The standard procedure to solve Eqs. (2)-(4) involves the "intermolecular" frame expansion in spherical harmonics of the correlation function, ${ }^{14}$ i.e.,

$$
g\left(R, \omega_{1}, \omega_{2}\right)=4 \pi \sum_{l_{1} l_{2} m} g_{l_{1} l_{2} m}(R) Y_{l_{1} m}\left(\omega_{1}\right) Y_{l_{2} \bar{m}}\left(\omega_{2}\right),
$$

where $\omega_{1}, \omega_{2}$ refer to the orientations of molecules 1,2 in a polar coordinates reference frame in which the $z$ axis lies along the vector joining the molecular centers $\mathbf{R}_{12}$ $\left(R=\left|\mathbf{R}_{12}\right|\right)$, or atomic sites, as was done in Ref. 7 .

The pair correlation function available from diffraction measurements is the atom-atom distribution function, $g_{\alpha \beta}(r)$, via $S_{\alpha \beta}(Q)$. Extracting site-site information from orientational correlations between molecules is, in principle, analytically feasible providing the coefficients of the spherical harmonics expansion are known. ${ }^{15}$ In $Q$ space, the sitesite correlation function is exactly defined in terms of Hankel transforms of the spherical harmonics and, for linear molecules, reads as follows: ${ }^{16}$

$$
\begin{aligned}
h_{\alpha \beta}(Q)= & (4 \pi)^{-3 / 2} \sum_{l_{1} l_{2} l} f\left(l_{1} l_{2} l\right) C\left(l_{1} l_{2} l ; 000\right) \\
& \times j_{l_{1}}\left(Q d_{1 \alpha}\right) j_{l_{2}}\left(Q d_{2 \beta}\right) h\left(l_{1} l_{2} l ; Q\right),
\end{aligned}
$$

where

$$
f\left(l_{1} l_{2} l\right)=i^{3 l_{1}+l_{2}}\left[\left(2 l_{1}+1\right)\left(2 l_{2}+1\right)(2 l+1)\right]^{1 / 2}
$$

$j_{l}(x)$ is the $l$ th spherical Bessel function, $\mathbf{d}_{i \alpha}\left(d_{i \alpha}=\left|\mathbf{d}_{i \alpha}\right|\right)$ is the vector displacement of the site $\alpha$ belonging to molecule $i$, from its center of mass, and

$$
h\left(l_{1} l_{2} l ; Q\right)=4 \pi i^{l} \int_{0}^{\infty} R^{2} h\left(l_{1} l_{2} l ; R\right) j_{l}(Q R) d R
$$

is the Hankel transform function of the spherical harmonic projection, $h\left(l_{1} l_{2} l ; R\right)$, of the total correlation function $[h(12)=g(12)-1]$ in a "laboratory-fixed" reference frame. ${ }^{17}$

This expression was successfully applied by Soper et al. in a recent work, ${ }^{18}$ in order to extract orientational correlation information from neutron diffraction data. Unfortunately, this procedure is somehow limited to moderately anisotropic systems for which the expansion is reasonably convergent. In a general case when molecular anisotropy plays a leading role, the expansion is poorly convergent if the full $g(12)$ is to be reconstructed, for instance, for an accurate evaluation of thermodynamic properties. ${ }^{19}$ In fact,

where 
TABLE I. Thermodynamic states of the systems considered in this work.

\begin{tabular}{lcc}
\hline \hline & $\mathrm{HCl}$ & $\mathrm{HI}$ \\
\hline$T(\mathrm{~K})$ & 297 & 253 \\
$\rho\left(\mathrm{g} / \mathrm{cm}^{3}\right)$ & 0.836 & 2.612 \\
$T^{*}$ & 1.5566 & 1.2650 \\
$\rho^{*}$ & 0.5749 & 0.8169 \\
\hline \hline
\end{tabular}

preliminary calculations performed by us on a model for $\mathrm{HCl}$ using Eq. (4) exhibited a poor convergence for the short and medium $Q$ range of $S_{\alpha \beta}(Q)$.

Let us recall that $g_{\alpha \beta}(r)$, on the other hand, is nothing but a constrained average over the angular variables of the orientational correlation function. Consequently, in practical terms, the way of computing the site-site correlation function requires going through a multidimensional numerical integration, which for linear molecules $\operatorname{read}^{5}$ as

$$
\begin{aligned}
g_{\alpha \beta}(r)= & \frac{1}{8 \pi} \int d \mathbf{R} d \cos \theta_{1} d \cos \theta_{2} d \phi_{12} g\left(\mathbf{R}, \theta_{1}, \theta_{2}, \phi_{12}\right) \\
& \times \delta\left[\mathbf{R}+\mathbf{d}_{2 \beta}-\mathbf{d}_{1 \alpha}-\mathbf{r}\right] .
\end{aligned}
$$

The constraints imposed by the $\delta$ function have been bypassed by means of the computational device ${ }^{20}$ sketched below. The numerical evaluation of $g_{\alpha \beta}(r)$ is performed in our calculation by means of a multidimensional Gaussian integration as follows, ${ }^{5}$

$$
\begin{aligned}
g_{\alpha \beta}(r)= & \frac{1}{n} \sum_{i=1}^{n_{r}} \sum_{k_{1}, k_{2}, j=1}^{n}\left\{w_{k_{1}} w_{k_{2}} \exp [-\beta u(12)\right. \\
& \left.\left.+\gamma(12)-B_{0}(12)\right]\left(\frac{R_{i}}{r}\right)^{2}\right\} \\
& \times \Delta R_{i} \delta\left(r-\Re_{\alpha \beta}\left(R_{i}, x_{k_{1}}, x_{k_{2}}, y_{j}\right)\right),
\end{aligned}
$$

where $w_{k_{i}}$ denotes the weights of Gauss-Legendre quadrature over the variables $x_{k_{i}}=\cos \theta_{i} .1 / n$ is the weight of Gauss-Chebyshev quadrature over the variable $y_{j}=\cos \phi_{12}$, with $n$ being the number of grid points in angular integration and $n_{r}$ is the number of grip points on the $R$ coordinate, which is tabulated in equal size intervals $\Delta r$, i.e., $R_{i}=i \cdot \Delta r \quad\left(i=1, \ldots, n_{r}\right)$. Obviously, $x_{k_{i}}$ and $y_{j}$ are, respectively, the roots of the Legendre polynomial $P_{n}(x)$ and the Chebyshev polynomial $T_{n}(y)$. The last factor in Eq. (14), $\left(R_{i} / r\right)^{2}$, is the ratio between volume elements of each reference frame, center-to-center and site-site frames or, in other words, the Jacobian of the reference frame transformation. $\mathfrak{R}_{\alpha \beta}\left(R_{i}, x_{k_{1}}, x_{k_{2}}, y_{j}\right)$ represents a generic atom-atom separation function that for heteronuclear molecules depends on the integration variables in the following way:

$$
\begin{aligned}
\mathfrak{R}_{a a}^{2}= & R_{i}^{2}+2 d_{1 a}^{2}-2\left[R_{i} d_{1 a} x_{k_{1}}-R_{i} d_{1 a} x_{k_{2}}\right. \\
& \left.+d_{1 a}^{2} \sqrt{1-x_{k_{1}}^{2}} \sqrt{1-x_{k_{2}}^{2}} y_{j}\right], \\
\mathfrak{R}_{a b}^{2}= & R_{i}^{2}+\left(d_{1 a}^{2}+d_{1 b}^{2}\right)-2\left[R_{i} d_{1 a} x_{k_{1}}+R_{i} d_{2 b} x_{k_{2}}\right. \\
& \left.-d_{1 a} d_{2 b} \sqrt{1-x_{k_{1}}^{2}} \sqrt{1-x_{k_{2}}^{2}} y_{j}\right],
\end{aligned}
$$

TABLE II. Potential parameters for the hydrogen halide liquid models considered in this work

\begin{tabular}{lccc}
\hline \hline & & \multicolumn{2}{c}{$\mathrm{HI}$} \\
\cline { 3 - 4 } & $\mathrm{HCl}$ & Model A & Model B \\
\hline$\varepsilon_{\mathrm{X}-\mathrm{X}} / k_{B}(\mathrm{~K})$ & 190.9 & 200.0 & 200.0 \\
$\varepsilon_{\mathrm{H}-\mathrm{H}} / k_{B}(\mathrm{~K})$ & 14.7 & 10.0 & 80.0 \\
$\varepsilon_{\mathrm{H}-\mathrm{X}} / k_{B}(\mathrm{~K})$ & $\sqrt{\varepsilon_{\mathrm{Cl}} \varepsilon_{\mathrm{H}}}$ & $\sqrt{\varepsilon_{\mathrm{I}} \varepsilon_{\mathrm{H}}}$ & 30.0 \\
$\sigma_{\mathrm{X}-\mathrm{X}}(\AA)$ & 3.47 & 4.05 & 4.05 \\
$\sigma_{\mathrm{H}-\mathrm{H}}(\AA)$ & 2.81 & 2.40 & 2.80 \\
$\sigma_{\mathrm{H}-\mathrm{X}}(\AA)$ & $\left(\sigma_{\mathrm{Cl}}+\sigma_{\mathrm{H}}\right) / 2$ & $\left(\sigma_{\mathrm{I}}+\sigma_{\mathrm{H}}\right) / 2$ & 3.0 \\
$l_{\mathrm{H}-\mathrm{X}}(\AA)$ & 1.30 & 1.61 & 1.61 \\
$\alpha\left(\AA^{3}\right)$ & 2.64 & $\cdots$ & $\cdots$ \\
$\mu(\mathrm{D})$ & 1.08 & $\cdots$ & $\cdots$ \\
$Q(\mathrm{~B})$ & 3.93 & $\cdots$ & $\cdots$ \\
\hline \hline
\end{tabular}

$$
\begin{aligned}
\Re_{b a}^{2}= & R_{i}^{2}+\left(d_{1 a}^{2}+d_{1 b}^{2}\right)+2\left[R_{i} d_{2 b} x_{k_{1}}+R_{i} d_{1 a} x_{k_{2}}\right. \\
& \left.+d_{1 a} d_{2 b} \sqrt{1-x_{k_{1}}^{2}} \sqrt{1-x_{k_{2}}^{2}} y_{j}\right], \\
\mathfrak{R}_{b b}^{2}= & R_{i}^{2}+2 d_{1 b}^{2}+2\left[R_{i} d_{2 b} x_{k_{1}}-R_{i} d_{1 a} x_{k_{2}}\right. \\
& \left.-d_{2 b}^{2} \sqrt{1-x_{k_{1}}^{2}} \sqrt{1-x_{k_{2}}^{2}} y_{j}\right] .
\end{aligned}
$$

It is worth to stress here that in Eq. (14) the molecular distribution function is evaluated through the closure given by Eq. (3), and not by resummation of the series expansion of $g(12)$, since in this way the most anisotropic component of $g(12)$ is treated exactly through the Boltzmann exponential in Eq. (3). The effect of the $\Delta R_{i} \delta\left(r-\Re_{\alpha \beta}\right)$ term in Eq. (14) implies that while stepping through all allowed $\cos \theta_{1}$, $\cos \theta_{2}$, and $\cos \phi_{12}$, and all distances $R_{i}$, the expression inside the curly brackets is evaluated, together with the distance between $\alpha-\beta$ sites $\left(r=\mathfrak{R}_{\alpha \beta}\right)$ and the appropriate $r$ bin of $g_{\alpha \beta}(r)$ is accordingly incremented.

\section{ON NEUTRON DIFFRACTION MEASUREMENTS: ISOTOPIC SUBSTITUTION METHOD}

From the experimental point of view, the molecular structure factor can be obtained by means of thermal neutron diffraction measurements,

$$
S_{M}(Q)=f_{1}(Q)+D_{M}(Q),
$$

where $f_{1}(Q)$ accounts for the intramolecular contribution and the intermolecular part is given by $D_{M}(Q)$, which for a heteronuclear diatomic fluid reads as

TABLE III. Compressibility factor and internal energy from both theory and MC simulation.

\begin{tabular}{llll}
\hline \hline & & \multicolumn{2}{c}{$\mathrm{HI}$} \\
\cline { 3 - 4 } & \multicolumn{1}{c}{$\mathrm{HCl}$} & \multicolumn{1}{c}{ Model A } & \multicolumn{1}{c}{ Model B } \\
\hline$(\beta U / N)_{\mathrm{MC}}$ & $-5.18 \pm 0.03$ & $-5.18 \pm 0.01$ & $-5.20 \pm 0.01$ \\
$(\beta U / N)_{\mathrm{RHNC}-\mathrm{VM}}$ & -5.11 & -5.13 & -5.24 \\
$(\beta P / \rho)_{\mathrm{MC}}$ & $-0.36 \pm 0.09$ & $2.46 \pm 0.07$ & $2.15 \pm 0.05$ \\
$(\beta P / \rho)_{\mathrm{RHNC}-\mathrm{VM}}$ & -0.54 & 2.76 & 1.90 \\
\hline \hline
\end{tabular}




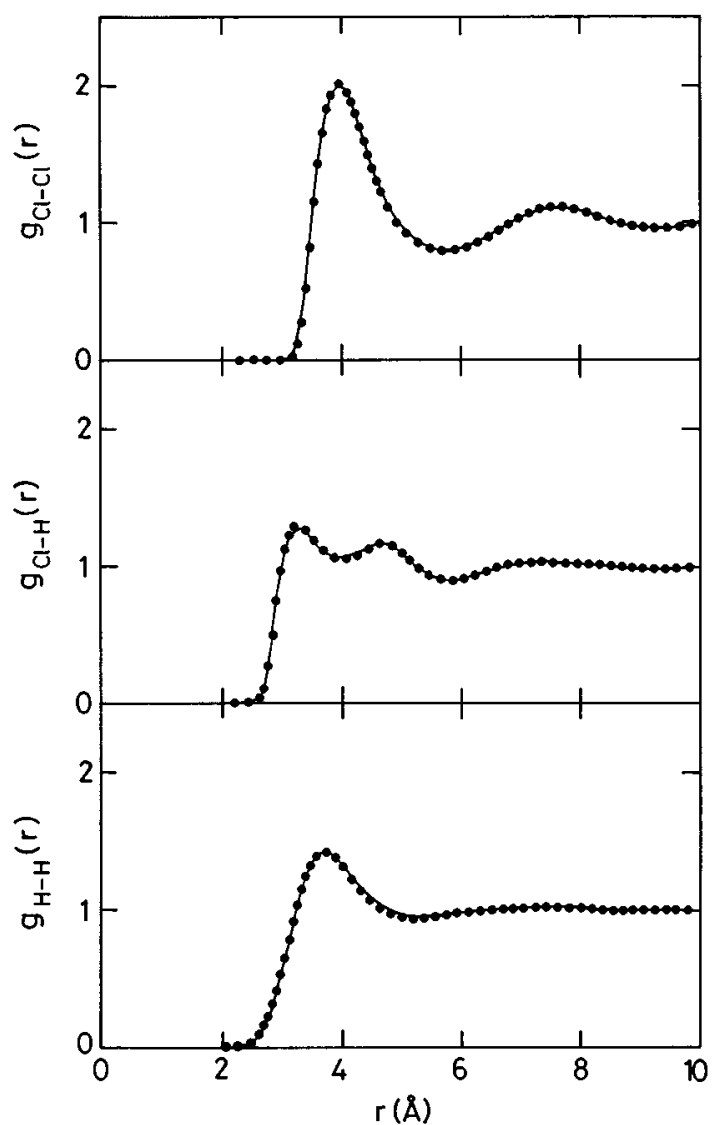

FIG. 1. Site-site pair distribution functions, $g_{\alpha \beta}(r)$, for liquid $\mathrm{HCl}$ at $T=297$ K. RHNC-VM (continuous line) versus MC simulations (solid circles).

$$
\begin{aligned}
D_{M}(Q)= & \frac{1}{b_{a}^{2}+b_{b}^{2}}\left\{b_{a}^{2}\left[S_{a a}(Q)-1\right]+b_{b}^{2}\left[S_{b b}(Q)-1\right]\right. \\
& \left.+2 b_{a} b_{b} S_{a b}(Q)\right\},
\end{aligned}
$$

with $b_{\alpha}$ being the neutron scattering lengths. Those individual components in which we are interested, may, in principle, be picked out from the composite function $D_{M}(Q)$. In order to determine all three $S_{\alpha \beta}(Q)$, three or more independent measurements have to be made by isotopic variation of the values $b_{\alpha}$. This method can only be achieved in a few favorable cases where the neutron scattering lengths change significantly between different isotopes. Therefore, since on hydrogen chloride both hydrogen and chlorine atoms can be isotope substituted $\left(b_{\mathrm{H}}=-0.374, \quad b_{\mathrm{D}}=0.667, \quad b^{35} \mathrm{Cl}\right.$ $=1.17$, and $\left.b^{37} \mathrm{Cl}=0.308 \times 10^{-12} \mathrm{~cm}\right)$, Soper et al. ${ }^{11}$ made use of the differencing method with $\mathrm{HCl}, \mathrm{DCl}$, ' $\mathrm{DH}$ ' Cl, $\mathrm{H}^{35} \mathrm{Cl}, \mathrm{H}^{37} \mathrm{Cl}$, and $\mathrm{D}^{\text {nat }} \mathrm{Cl}$ diffraction patterns, in order to obtain the three partial structure factors. In the case of hydrogen iodide, where only the hydrogen atom can be labeled, the three different partial structure factors were obtained by means of neutron diffraction experiments on DI, HI, and equimolar mixture of HI and DI ('DH'I). ${ }^{13}$ Note that X-ray diffraction measurements would, in any event, give virtually only $g_{\mathrm{Cl}-\mathrm{Cl}}(r)$ and $g_{\mathrm{I}-\mathrm{I}}(r)$.

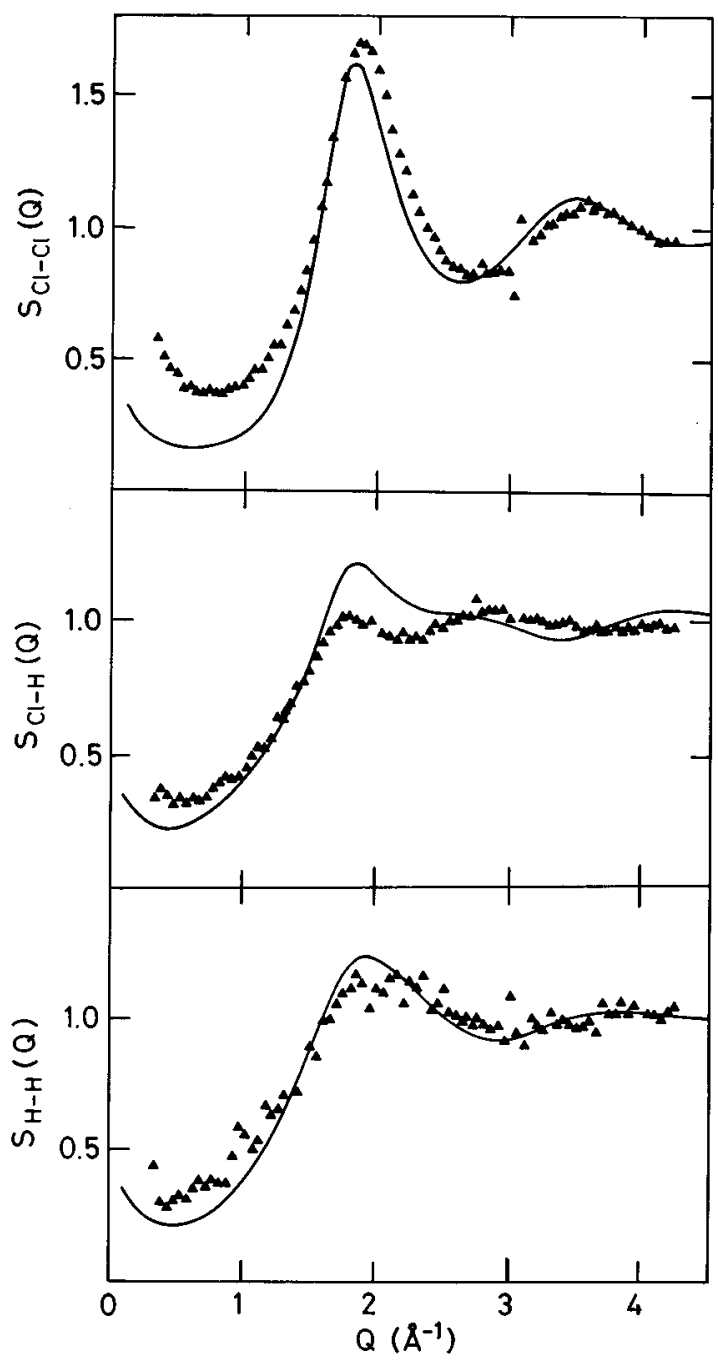

FIG. 2. Atom-atom structure factors, $S_{\alpha \beta}(Q)$, for liquid $\mathrm{HCl}$ at $T=297 \mathrm{~K}$. RHNC-VM (continuous line) versus neutron diffraction data (solid triangles) from Ref. 11.

In Table I the thermodynamic state parameters considered in our work, in accordance with the available experimental data, are summarized.

\section{RESULTS AND CONCLUSIONS}

We have applied the RHNC-VM approximation to three different fluid models, one corresponding to $\mathrm{HCl}$ and other two models representing HI. The interaction potential model used for describing $\mathrm{HCl}$ consists, roughly speaking, of a twocenter Lennard-Jones diatomic with a permanent dipole $\boldsymbol{\mu}$, a linear quadrupole moment, $\mathbf{Q}$, and $\boldsymbol{\alpha}$, the isotropic polarizability located at the center of mass, which is treated by means of an effective dipole moment. ${ }^{2}$ For an explicit formula of this potential model, the reader is referred to Eq. (1) of Ref. 2. Values of the potential parameters for each model can be found in Table II. Due to the absence of intermolecular potentials in the literature for describing a HI liquid, we have constructed two 2CLJ potential models ad hoc, which will be denoted model $\mathrm{A}$ and model $\mathrm{B}$. The main difference between them lies in the fact that cross terms $H-X$ for 


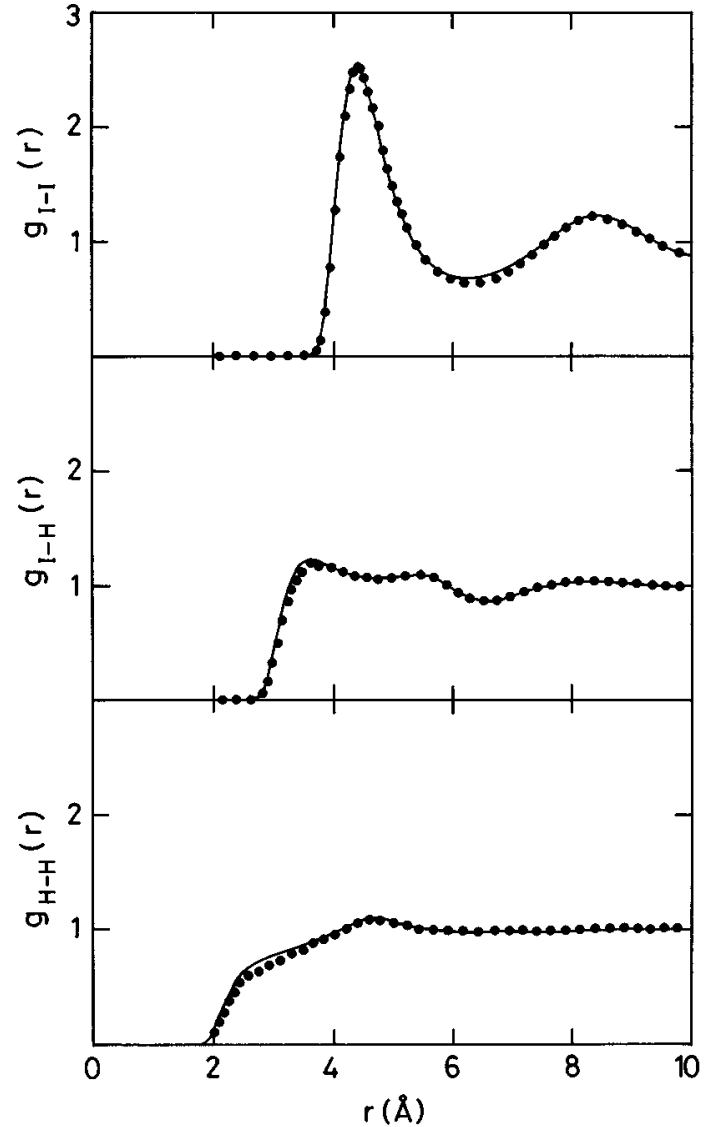

FIG. 3. Site-site pair distribution functions, $g_{\alpha \beta}(r)$, for liquid HI at $T=253$ $\mathrm{K}$. RHNC-VM/model A (continuous line) versus MC simulations (solid circles).

model B do not follow the Lorentz-Berthelot mixing rules [Eq. (9)]. The choice of the interaction parameters was guided by two empirical criteria. First, the net interaction obtained by addition of the three atom-atom potentials is chosen to add up to a value consistent with the experimental critical point of $\mathrm{HI} .{ }^{13}$ The interaction range parameters are defined according to standard values for $\mathrm{H}$ and I (Refs. 7,13, and references therein). The tuning of the parameters has been done so that the site-site distribution functions reproduce qualitatively the profile of the functions extracted from experimental partial structure factors (Fig. 5 of Ref. 13). The nonadditivity was also taken into account for the reference system calculation, i.e., when defining the cross-interaction hard sphere diameters. Particularly, cross terms were calculated according to an extension of Eq. (7),

$$
d_{a b}=\sigma_{a b} g\left(\gamma^{\prime}\right)\left[25-\ln \left(k_{B} T / \varepsilon_{a b}\right)\right]\left[25+\ln \left(\rho \sigma_{a b}^{3}\right)\right],
$$

instead of the use of mixing rules $\left(\gamma^{\prime}=\sigma_{a b} / \sigma_{a}\right)$.

The molecular integral equation was solved in the RHNC-VM approximation using 35 coefficients [i.e., $l_{1}, l_{2} \leqslant 4$ for $g_{l_{1} l_{2} m}(R)$ in Eq. (10)]. The number of grid points in angular integration used in the evaluation of the multidimensional integrals [Eq. (14)] was set to $n=30$ and the spacing in $R$ to $\Delta r=0.02 \sigma_{a}$ with 512 mesh points. RHNC-VM calculations and Monte Carlo (MC) computer

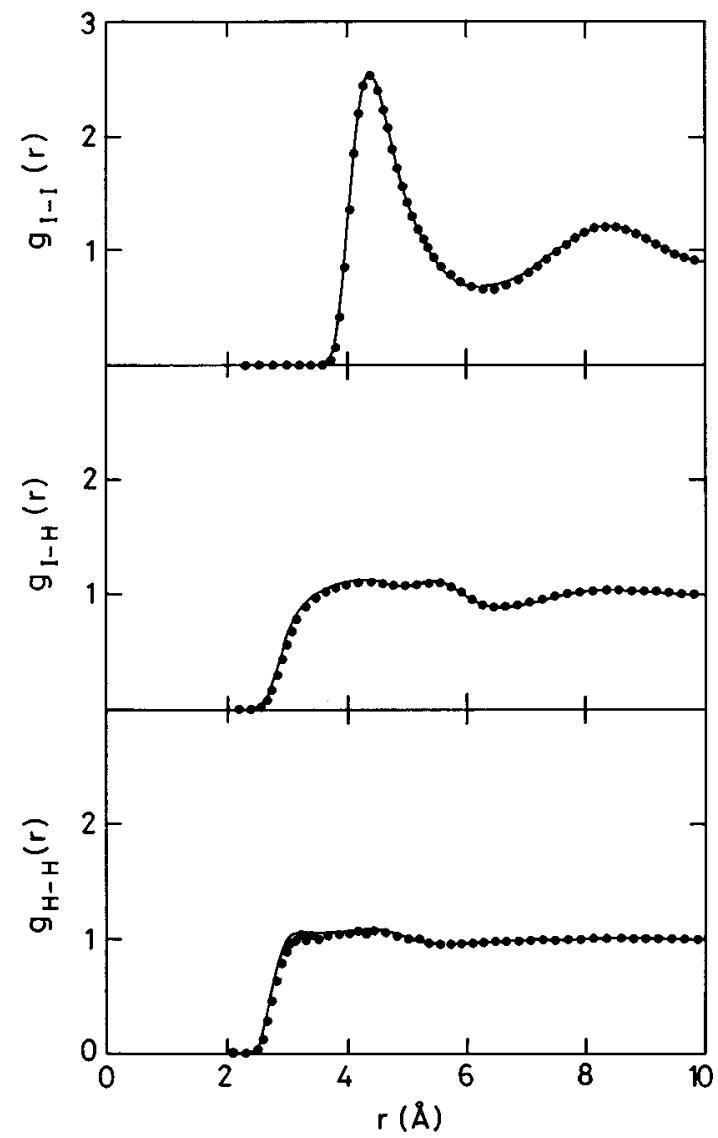

FIG. 4. Site-site pair distribution functions, $g_{\alpha \beta}(r)$, for liquid $\mathrm{HI}$ at $T=253$ $\mathrm{K}$. RHNC-VM/model B (continuous line) versus MC simulations (solid circles).

simulations were performed at the thermodynamic states summarized in Table I, for the intermolecular potential models whose parameters are compiled in Table II. Thermodynamic properties from theory and MC simulation are collected in Table III.

The results for $\mathrm{HCl}$ are plotted in Figs. 1 and 2, and those corresponding to $\mathrm{HI}$ are presented in Figs. 3-5. Figures 1,3 , and 4 display the RHNC-VM site-site pair distribution function versus MC results. All of them show the extremely good agreement between theory and simulation, both for $\mathrm{HCl}$ (Fig. 1) and HI, model A (Fig. 3) and model B (Fig. 4), corroborating previous conclusions about the goodness of the RHNC-VM approach. ${ }^{7}$ In Figs. 2 and 5 one can compare the theoretical partial structure factors with those extracted from neutron diffraction data. As already known, the $\mathrm{X}-\mathrm{X}$ correlation $(X=$ halogen atom), which are essentially coincident with the center-to-center correlations, are those better described, though with some discrepancies in the intensity of the first peak. As to the molecular orientation-dependent terms, $\mathrm{H}-\mathrm{H}$ and $\mathrm{X}-\mathrm{H}$, the situation is less satisfactory, again due to the inadequacies of the interaction models. Moreover, it is worthwhile stressing that the two potential models proposed for the description of the structure of HI fluid go into the right direction (see Fig. 5). For instance, the accordance between our theoretical results and experimental $S_{\mathrm{I}-\mathrm{H}}(Q)$ is better than that of the orientationally uncorrelated model 


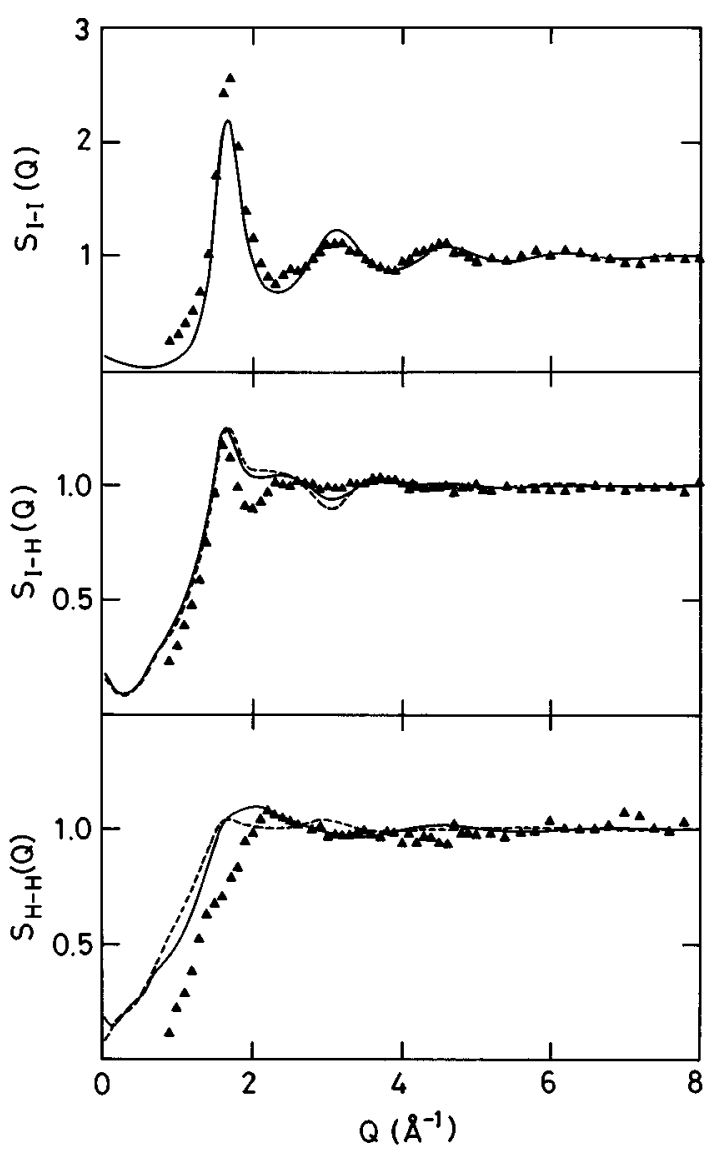

FIG. 5. Atom-atom structure factors, $S_{\alpha \beta}(Q)$, for liquid $\mathrm{HI}$ at $T=253 \mathrm{~K}$. RHNC-VM/model A (dashed line), RHNC-VM/model B (continuous line) versus neutron diffraction data (solid triangles) from Ref. 13. $S_{\mathrm{III}}(Q)$ from both models are indistinguishable at the scale of the figure.

used in Ref. 13 (see Fig. 4 in that reference). Additionally, the nonadditive model (model B) appears to be slightly more suitable to account for the first peak present in the $S_{\mathrm{H}-\mathrm{H}}(Q)$ experimental pattern. Both model A and the abovementioned uncorrelated model deviate considerably within this region. It is obvious that an essentially different approach must be undertaken if the limitations of these potential models are to be overcome. In this regard, the site-site angular-dependent interaction potentials introduced in recent years by Rodger, Stone, and Tildesley for liquid halogens, ${ }^{21}$ might offer an interesting alternative. These potential models, though formally more complicated that the $2 \mathrm{CLJ}$, are still suitable for the integral equation treatment presented in this work, even if a new parametrization of the reference system might be required.
In summary, an efficient procedure for computing the atomic structure factors from integral equation calculations has been presented and explored with success in the three simple models of heteronuclear diatomic fluids. Its extension to nonlinear molecules implies the use of rotational invariant expansions and five-fold multidimensional integrations. Although the computational procedure is considerably more involved, it is still feasible, and work on this is currently in progress. ${ }^{22}$

\section{ACKNOWLEDGMENTS}

The authors would like to thank Dr. A. K. Soper and Dr. F. Lado for their very helpful comments. This research was financially supported by the Spanish Dirección General de Investigación Científica y Técnica (DGICYT) under Grant No. PB91-0110.

${ }^{1}$ E. Lomba, C. Martín, M. Lombardero, and J. A. Anta, J. Chem. Phys. 96, 6132 (1992); M. Lombardero, C. Martín, and E. Lomba, ibid. 97, 2724 (1992); C. Martín, M. Lombardero, and E. Lomba, ibid. 98, 6465 (1993).

${ }^{2}$ C. Martín, E. Lomba, M. Lombardero, F. Lado, and J. S. Høye, J. Chem. Phys. 100, 1599 (1994).

${ }^{3}$ M. Lombardero, C. Martín, and E. Lomba, Mol. Phys. 81, 1313 (1994).

${ }^{4}$ F. Lado, S. M. Foiles, and N. W. Ashcroft, Phys. Rev. A 28, 2374 (1983).

${ }^{5}$ F. Lado, Mol. Phys. 47, 283, 299 (1982).

${ }^{6}$ L. Verlet, Mol. Phys. 41, 183 (1980); S. Labík, A. Malijevský, and W. R. Smith, ibid. 73, 87, 495 (1991).

${ }^{7}$ C. Martín, M. Lombardero, M. Alvarez, and E. Lomba, J. Chem. Phys. 102, 2092 (1995).

${ }^{8}$ S. Murad, K. E. Gubbins, and J. G. Powles, ibid. 40, 253 (1980).

${ }^{9}$ I. R. McDonald, S. F. G. Shea, D. G. Bounds, and M. L. Klein, J. Chem. Phys. 72, 5710 (1980).

${ }^{10}$ M. L. Klein and I. R. McDonald, Mol. Phys. 42, 243 (1981).

${ }^{11}$ A. K. Soper and P. A. Egelstaff, Mol. Phys. 42, 399 (1981).

${ }^{12}$ J. G. Powles, E. K. Osae, J. C. Dore, and P. Chieux, ibid. 43, 1051 (1981).

${ }^{13}$ C. Andreani, M. Nardone, F. P. Ricci, and A. K. Soper, Phys. Rev. A 46, 4709 (1992).

${ }^{14}$ Chapter 12, in Theory of Simple Liquids, by J.-P. Hansen and I. R. McDonald (Academic, London, 1986).

${ }^{15}$ J. S. Høye and G. Stell, J. Chem. Phys. 66, 795 (1979).

${ }^{16}$ For details, see Sec. 3.2, Chap. 3, in Theory of Molecular Fluids. Volume 1: Fundaments, by C. G. Gray and K. E. Gubbins (Clarendon, Oxford, 1984). Notation conventions therein have been followed in this work.

${ }^{17}$ The general relation between the two sets of coefficients, coming from the "intermolecular" frame expansion and the "laboratory-fixed" frame expansion, is given by Eq. (12.2.8) in Ref. 14.

${ }^{18}$ A. K. Soper, C. Andreani, and M. Nardone, Phys. Rev. E 47, 2598 (1993).

${ }^{19} \mathrm{~F}$. Lado (private communication)

${ }^{20}$ Streamlines of this procedure were suggested to us by A. K. Soper in connection to his own work in Ref. 18.

${ }^{21}$ P. M. Rodger, A. J. Stone, and D. J. Tildesley, Mol. Phys. 63, 173 (1988); Chem. Phys. Lett. 145, 365 (1988).

${ }^{22}$ F. Lado, E. Lomba, and M. Lombardero, J. Chem. Phys. (in press). 